\title{
Effects of a Home-Based Family-Centred Early Habilitation Program on Neurobehavioural Outcomes of Very Preterm Born Infants: A Retrospective Cohort Study
}

\author{
Michela Poggioli, ${ }^{1}$ Fabrizio Minichilli, ${ }^{2}$ Tiziana Bononi, ${ }^{1}$ Pasquina Meghi, ${ }^{3}$ \\ Paolo Andre, ${ }^{4}$ Alessandra Crecchi, ${ }^{5}$ Bruno Rossi, ${ }^{5}$ Maria Chiara Carboncini, \\ Alberto Ottolini, ${ }^{1}$ and Luca Bonfiglio ${ }^{5}$ \\ ${ }^{1}$ Unità Operativa Neuropsichiatria Infanzia e Adolescenza (UONPIA), ASST Fatebenefratelli e Sacco, 20157 Milano, Italy \\ ${ }^{2}$ Unit of Environmental Epidemiology, Institute of Clinical Physiology, National Council of Research, 56124 Pisa, Italy \\ ${ }^{3}$ Unità Operativa Neuropsichiatria Infanzia e Adolescenza (UONPIA), ASST Grande Ospedale Metropolitano Niguarda, \\ 20162 Milano, Italy \\ ${ }^{4}$ Department of Medicine, Surgery and Neuroscience, University of Siena, 53100 Siena, Italy \\ ${ }^{5}$ Department of Translational Research on New Technologies in Medicine and Surgery, School of Physical Medicine and Rehabilitation, \\ University of Pisa, 56126 Pisa, Italy
}

Correspondence should be addressed to Luca Bonfiglio; bubobis@gmail.com

Received 22 July 2016; Revised 16 November 2016; Accepted 28 November 2016

Academic Editor: Bruno Poucet

Copyright (C) 2016 Michela Poggioli et al. This is an open access article distributed under the Creative Commons Attribution License, which permits unrestricted use, distribution, and reproduction in any medium, provided the original work is properly cited.

\begin{abstract}
Preterm children have an increased risk of neurodevelopmental impairments which include psychomotor and language retardation. The objectives of the present retrospective cohort study were to examine the effects of an individually adapted, home-based, and family-centred early developmental habilitation program on neurodevelopmental and behavioural outcomes of very preterm children compared with a standard follow-up at 2 years' corrected age. Enrolled infants were retrospectively assigned to the intervention group (61 subjects) or to the control group (62 subjects) depending on whether they had or had not carried out a home-based family-centred early developmental habilitation program focused on environmental enrichment, parent-guided environmental interaction, and infant development. Developmental outcome was assessed for both groups at 24 months' corrected age using the Bayley Scales of Infant Development 2nd Edition. Intervention significantly improved both cognitive and behavioural outcomes. In addition, males had significantly lower scores than females either before or after treatment. However, the treatment was effective in both genders to the same extent. In conclusion, a timely updated environment suitable to the infant's developmental needs could provide the best substrate where the parent-infant relationship can be practised with the ultimate goal of achieving further developmental steps.
\end{abstract}

\section{Introduction}

A baby born alive before completion of the 37 th week of pregnancy is defined as preterm (WHO). Fifteen million infants were born preterm in 2010, which has major societal implications [1]. Moreover, the fact that the rate of preterm births remains unchanged or even slightly increases on a yearly basis means that, considering an annual increase in the global population worldwide, the global number of preterm births increases every year [1].
Several subcategories of preterm infants are identified on the basis of both gestational age ( 32 to $<37$ weeks: moderate to late preterm; 28 to $<32$ weeks: very preterm; $<28$ weeks: extremely preterm) and weight at birth $(1500$ to $<2500 \mathrm{~g}$ : low birthweight, LBW; 1000 to $<1500$ g: very low birthweight, VLBW; <1000 g: extremely low birthweight, ELBW), reflecting increasing degrees of prematurity. This classification is justified by the fact that the risk of death within the first month of life (about 1 million in 2010 for direct complications of preterm birth) and the risk of developing subsequent 
cognitive and motor impairments in survivors grow with the degree of prematurity [1].

Children who survive (about 13 million worldwide, but with an uneven geographical distribution depending on countries' income differences) [1] have an increased risk of neurodevelopmental impairments which include psychomotor and language developmental delays, vision and hearing loss, cerebral palsy, school learning difficulties, and mental disorders during adulthood.

Therefore, a portion of babies born preterm may end up representing a cost in either economic (due to neonatal intensive care, continuing healthcare, and special educational needs) or social and family terms, both in the short and medium-long term. From this point of view, for some years now, early (i.e., within the first year of life) intervention programs have been conceived that, as far as possible, are able to prevent and/or mitigate motor and cognitive impairments due to prematurity. This early intervention is needed to try to harness the most favourable time window to the entry into play of plastic capacities of the brain, which are expected to have a potential role of protection from and/or restoration of neurological impairments [2-4].

The idea is that appropriate stimulation and/or perceptions may pose robust "neurobiological" bases for the harmonious development of the baby's motor, cognitive, and behavioural profile, by fostering the development of brain connections [5] and, consequently, functional networks. The hope is that it might be possible to arrive at a real action of brain-moulding by modulating environmental demands addressed to the child $[6,7]$.

The heterogeneity of these programs in terms of either type (focused on the infant development and/or parent-infant relationship) or frequency and duration of the intervention has made it difficult to draw to date any firm conclusions on their real effectiveness [8]. Nevertheless, recent metaanalysis studies $[8,9]$ have been able to confirm a significant improvement of cognitive and/or motor outcomes in the short to medium term, that is, within the infant- (zero to $<$ three years) and/or the preschool age (three to $<$ five years), in particular for those programs that are focused on both the infant development and the parent-infant relationship. On the contrary, they have not demonstrated significant or unambiguous effects on cognitive and motor outcomes in the long term, that is, within the school age (five to $<17$ years) or adulthood.

The objectives of the present retrospective cohort study were (a) to examine the effects of an individually adapted, home-based, and family-centred early developmental habilitation program on neurodevelopmental and behavioural outcomes of very preterm born children compared with standard follow-up at 2 years' corrected age and (b) to identify possible treatment-modifying factors.

Distinctive elements of the present retrospective cohort study with respect to previous studies involving early developmental interventions of a similar type (i.e., studies comprising two phases of treatment, in hospital and after discharge, the latter carried out at home for a period of at least 12 months) were that (a) the main focus of the program was directed not only to the infant development and parent-infant relationship, but also to a properly enriched and changing home environment to be provided to the child, and (b) the frequency of health professionals outpatient visits (i.e., the total number of visits per treatment duration) was lower, since those who were delegated to carry out daily the homebased program were the children's parents themselves.

\section{Methods}

2.1. Participants and Study Design. One hundred and twentythree preterm infants constituted the study sample. They were recruited from a retrospective chart review of preterm born infants admitted in the Neonatal Intensive Care Unit (NICU) of the Macedonio Melloni Hospital-Fatebenefratelli e Oftalmico (Milan, Italy) between 1998 and 2006. Selection criteria included gestational age at birth of $<32$ weeks (very preterm infant, VPI) and/or weight at birth $<1500 \mathrm{~g}$ (very and extremely low birth weight, VLBW and ELBW). Exclusion criteria included congenital or chromosomal abnormalities, congenital or acquired infections, known prenatal brain lesions, periventricular leukomalacia, intraventricular hemorrhages $>$ II grade, neonatal seizures, and peripheral nervous system disorders. As a consequence, cases with high risks of developing cerebral palsy (CP) were excluded, since the aim of our study was purely focused on delayed brain maturation rather than brain injuries.

Enrolled infants were retrospectively assigned to two groups depending on whether they had (intervention group, 61 subjects) or had not (control group, 62 subjects) carried out the outpatient phase of the intervention program. After an inpatient phase of the program, which started before discharge from the NICU and was common to either group, the intervention group received a home-based family-centred early developmental habilitation program (see below for further information), whilst the control group received the standard pediatric and neurological follow-up guaranteed by the community health services (consisting in a "wait-andsee" approach involving active monitoring of the infant over the first 12-18 months' corrected age).

Developmental outcome was then assessed for both groups at 24 months' corrected age using the Bayley Scales of Infant Development 2nd Edition (BSID-II) [10]. This is a norm-referenced test that yields standardised scores (mean, 100; SD, 15; range, 50 to 150) for cognitive (mental development index, MDI), motor (psychomotor development index, PDI), and behavioural development (behavioural rating scale, BRS).

2.2. Intervention. The intervention consisted in an individually adapted early developmental intervention program (EDIP). This program, while inspired by the principles of the Newborn Individualized Developmental Care and Assessment Program (NIDCAP) (see $[11,12]$ for reviews), did not involve the full and exclusive implementation of NIDCAP.

The EDIP consisted in two phases, the inpatient and the outpatient phase. The former began in the NICU with daily sessions from birth to discharge and was the same for both the intervention and the control group, since it was provided to all infants participating in the study as the standard NICU 
care program. The latter, according to the institution's policy, was offered to all families at discharge and consisted in a fixed follow-up program which provided for regular assessment and counseling sessions at $1,3,6,9$, and 12 months of corrected age and/or at acquired ambulation (i.e., approximately 18 months of corrected age). As an option, in selected cases, some additional meetings could be added on a need-oriented basis, so that the final average number of visits was 8 (range 5-11). In each follow-up session the development of the infant was checked and parents were instructed about how to carry out a home-based intervention program appropriate to the child's age until the next examination.

However, since some families did not join the postdischarge phase for logistical and/or personal reasons, the recruitment of the study groups took place retrospectively based on the actual participation of the subjects. In particular, only those subjects who had completed the entire program (i.e., subjects who completed all the planned follow-up sessions) were recruited in the intervention group, whilst subjects who had not carried out the outpatient phase were recruited in the control group. In this way, 61 cases and 62 controls were recruited.

2.2.1. Inpatient Phase. During hospitalization, all subjects participating in the study received the standard NICU care program which provided for all of the following measures/steps: restraint of excessive environmental stimuli, postural limits ("nesting," "holding," and "wrapping"), nonnutritive sucking, skin-to-skin contact (Kangaroo Care), and tactile stimulation (Gentle Touch). This phase was conducted by both nursing staff and parents themselves educated by a physiotherapist. All subjects, between the 35th and the 40th week of corrected age, were then submitted to a predischarge evaluation of the "synactive" type (i.e., based on the evaluation of the five functional subsystems described in her "synactive theory" by Als [13]: the autonomic system, the motor system, the state organizational system, the attentional-interactive system, and the self-regulatory system) by a dedicated staff composed of a child neurologist and a neonatologist.

2.2.2. Outpatient Phase. In follow-up sessions conducted by a pediatric physiotherapist after discharge up to three months' corrected age, a combined/mixed evaluation approach was followed, both of the classical "synactive" type and of the "ecological-transactional" type (i.e., based on the observation of the development of a functional use of motor skills, of the infant-parent interaction, and of the behavioural modulation). From the 4th month of corrected age until gait acquisition, the "synactive" evaluation was replaced by the "ecological-transactional" evaluation.

Each session of evaluation was followed by the formulation of care and habilitative advice for parents, aiming at achieving a child's developmental milestones, whose application and effectiveness would be checked in the subsequent/following evaluation.

The home-based and family-centred habilitation program, which was to be conducted by parents themselves, basically included the following four lines of intervention: motor, relational, environmental, and transactional.
On the motor plane, within the first three months of corrected age, the intervention was focused on the practices of postural containment ("nesting," "holding," and "wrapping"), aimed at maintaining a curled position of the infant, so as to promote the right adjustment of his/her postural tone. Subsequently, the scope of the intervention widened to promote in children adaptive responses to the environment, eye-contact with faces and objects ("engaging visual fixation and pursuit"), reaching/grasping/manipulation of objects, the choice of an autonomous mode of moving about (environmental navigation), the right timing of achievement of both standing and walking (e.g., by advising against the premature use of baby walker), autonomy in eating, collaboration in dressing, and the inconsistent staff-dependent inclusion on external water activities (pool activities).

Regarding the relational plane, the intervention extended from the creation of the parental bond-through skin-to-skin contact (Kangaroo Care), tactile stimulation (Gentle Touch), infant massage, contact gaze, and social interactions (smile, warble, and vocalization) - to the beginning of both the separation process from the adult/parents and the building of peer relationships, including the inconsistent staff-dependent access to child educational centres ( $>10$ months of corrected age).

On the environmental plane, offering a highly stimulating home environment, which had to be continuously variable/changed according to the child's developmental needs, was crucial. This was ensured through the creation of a dedicated space (e.g., at least at an early stage, by putting down a play-mat on the floor) where the child could be progressively involved in more active and complex play/motor activities (see Table 1) for increasing periods of time (e.g., from 15/20 minutes twice a day at 3 months c.e., to $45 / 60$ minutes twice a day at 6 months c.e., etc.). In this context, the child was free to experience and explore, thus increasing his/her autonomy and self-esteem, but, at the same time, becoming aware of his/her own limits and, thus, learning to experience related frustrations.

From all the above, it is clear how this approach places parents at the centre of the program, who, accompanied/guided and supported by the staff/team, are the protagonists of the promotion of their own child development. Therefore, hints by the team are targeted to raise parents' awareness to detect and interpret the signs of stress/discomfort and comfort/control of their child (conceptualized as "weak points" and "strength points," resp.), in order to help them to adjust their care intervention to support and stimulate their child's development, thus promoting both the emergence of motor skills and the modulation of the child's behaviour.

As a result, this promotes and consolidates parent-child interactions, thus creating the premises for the establishment of the so-called "affective tuning" between parents and child (also known as "parental bonding"), from which both parts derive mutual benefit and satisfaction (parent-infant transactional patterns/coregulation).

The program came to an end with the achievement of the child's ambulation (i.e., within approximately 18 months' corrected age). At 24 months of corrected age, all subjects afferent to the study (belonging to both the control group and 
TABLE 1: Training, differentiated by corrected age, of postural control, motor, and play activities, and environmental interactive skills.

\begin{tabular}{|c|c|c|c|}
\hline 3 mths c.e. & $6 \mathrm{mths}$ c.e. & 9-12 mths c.e. & $12-18$ mths c.e. \\
\hline $\begin{array}{l}\text { Maintaining a stable supine position } \\
\text { with both hands on the midline }\end{array}$ & Rolling over & Hand-walking, cruising & Walking without support \\
\hline Static and dynamic side-lying & Sitting & Using tricycle without pedals & Exploring environment \\
\hline $\begin{array}{l}\text { Maintaining a stable prone position } \\
\text { with or without axillary support }\end{array}$ & Crawling, bottom-shuffling & Standing & Trying to eat autonomously \\
\hline $\begin{array}{l}\text { Naturally rewarding multisensory } \\
\text { experiences }\end{array}$ & $\begin{array}{l}\text { Fetching attractive toys and } \\
\text { bringing them to mouth }\end{array}$ & $\begin{array}{l}\text { Free to experience standing up } \\
\text { and falling down }\end{array}$ & Taking part in dressing \\
\hline Eye contact, eye pursuit & & Emptying/filling containers & $\begin{array}{l}\text { Opportunities to socialize with } \\
\text { peers }\end{array}$ \\
\hline $\begin{array}{l}\text { Reaching, grasping, and handling } \\
\text { (rattles, colourful keys, puppets, etc.) }\end{array}$ & & Building activities & \\
\hline $\begin{array}{l}\text { Eye contact and social interaction } \\
\text { (smiling, vocalizing, lalling, etc.) }\end{array}$ & & Cause-and-effect toy playing & \\
\hline
\end{tabular}

TABLE 2: Comparisons between intervention and control groups on the basis of both demographic and clinical variables.

\begin{tabular}{|c|c|c|c|c|}
\hline & Categories & Intervention group & Control group & $p$ value \\
\hline Total number & & 61 & 62 & \\
\hline \multirow{2}{*}{ Gender } & Male & $34(56 \%)$ & $27(44 \%)$ & \multirow{2}{*}{0.176} \\
\hline & Female & $27(44 \%)$ & $35(56 \%)$ & \\
\hline Socioeconomic level & & $2.47 \pm 0.52$ & $2.5 \pm 0.72$ & 0.818 \\
\hline GA & & $209.9 \pm 1.78$ & $217.89 \pm 1.79$ & 0.002 \\
\hline BW & & $1245.25 \pm 37.20$ & $1422.74 \pm 47.69$ & 0.004 \\
\hline \multirow{2}{*}{ BW classification } & VLBW & $46(75 \%)$ & $52(84 \%)$ & \multirow{2}{*}{0.244} \\
\hline & ELBW & $15(25 \%)$ & $10(16 \%)$ & \\
\hline \multirow{2}{*}{ Size versus GA } & AGA & $48(79 \%)$ & $50(81 \%)$ & \multirow{2}{*}{0.787} \\
\hline & SGA & $13(21 \%)$ & $12(19 \%)$ & \\
\hline $\mathrm{HC}$ & & $26.95 \pm 0.30$ & $28.26 \pm 0.34$ & 0.005 \\
\hline \multirow{2}{*}{ Apgar score } & $1^{\prime} \mathrm{AS}$ & $6 \pm 0.29$ & $5.72 \pm 0.33$ & 0.528 \\
\hline & $5^{\prime} \mathrm{AS}$ & $7.95 \pm 0.17$ & $8.02 \pm 0.17$ & 0.770 \\
\hline \multirow{3}{*}{$\mathrm{CU}$} & Norm & $26(42 \%)$ & $36(58 \%)$ & \multirow{3}{*}{0.218} \\
\hline & Abnorm & $23(38 \%)$ & $16(26 \%)$ & \\
\hline & Ivh I, II & $12(20 \%)$ & $10(16 \%)$ & \\
\hline \multirow{2}{*}{ BPD } & No & $50(82 \%)$ & $59(95 \%)$ & \multirow{2}{*}{0.008} \\
\hline & Yes & $11(18 \%)$ & $3(5 \%)$ & \\
\hline $\mathrm{MV}$ & & $7.58 \pm 1.27$ & $5.53 \pm 1.03$ & 0.178 \\
\hline
\end{tabular}

GA: gestational age (days); BW: birth weight (g); VLBW: very low birth weight; ELBW: extremely low birth weight; AGA: appropriate for gestational age; SGA: small for gestational age; HC: head circumference (cm); 1'-AS: 1-minute Apgar score; $5^{\prime}$-AS: 5-minute Apgar score; CU: cranial ultrasound; Ivh: intraventricular hemorrhage; BPD: bronchopulmonary dysplasia; MV: mechanical ventilation (days). For quantitative variables, mean values \pm standard error of mean are reported. For qualitative variables, both absolute numbers and percentages are reported. When comparison between groups is significant, $p$ value is shown in bold.

the intervention group) were submitted to the final outcome evaluation by administering the BSID-II.

2.3. Statistical Analysis. The following variables were taken into consideration: MDI, PDI, and BRS, according to the BSID-II (as outcome measures); and days of gestational age (GA), birth weight (BW), categories of preterm infants on the basis of BW (VLBW and ELBW), categories on the basis of size versus GA (appropriate for GA, AGA, and small for G, SGA), head circumference (HC), 1-minute Apgar score
$\left(1^{\prime}-\mathrm{AS}\right)$ and 5-minute Apgar score (5'-AS), levels of severity on the basis of cranial ultrasound (CU) reports, presence or absence of bronchopulmonary dysplasia (BPD), and days of mechanical ventilation (MV) (as possible treatmentmodifying factors).

The statistical comparison between intervention and control groups on the basis of the observed dependent variables was performed using Student's $t$-test (for comparison between means) and Pearson's chi-squared test (for comparison between proportions) (Table 2). This is in order 
TABLE 3: Mutual relationships between observed clinical variables (quantitative variables) and outcome measures.

\begin{tabular}{|c|c|c|c|c|c|c|c|c|c|}
\hline & MDI & PDI & BRS & $\overline{\mathrm{GA}}$ & BW & $\mathrm{HC}$ & $1^{\prime}-\mathrm{AS}$ & $5^{\prime}-\mathrm{AS}$ & $\mathrm{MV}$ \\
\hline MDI & 1.0000 & & & & & & & & \\
\hline PDI & $\begin{array}{l}0.4375 \\
\mathbf{0 . 0 0 0 1}\end{array}$ & 1.0000 & & & & & & & \\
\hline BRS & $\begin{array}{c}0.6342 \\
\mathbf{0 . 0 0 0 0}\end{array}$ & $\begin{array}{l}0.4904 \\
\mathbf{0 . 0 0 0 0}\end{array}$ & 1.0000 & & & & & & \\
\hline GA & $\begin{array}{c}-0.0007 \\
1.0000\end{array}$ & $\begin{array}{c}-0.0542 \\
1.0000\end{array}$ & $\begin{array}{c}-0.2081 \\
1.0000\end{array}$ & 1.0000 & & & & & \\
\hline BW & $\begin{array}{c}-0.0180 \\
1.0000\end{array}$ & $\begin{array}{l}0.0347 \\
1.0000\end{array}$ & $\begin{array}{c}-0.0549 \\
1.0000\end{array}$ & $\begin{array}{c}0.6561 \\
\mathbf{0 . 0 0 0 0}\end{array}$ & 1.0000 & & & & \\
\hline $\mathrm{HC}$ & $\begin{array}{l}0.0748 \\
1.0000\end{array}$ & $\begin{array}{c}-0.0216 \\
1.0000\end{array}$ & $\begin{array}{c}0.0131 \\
1.0000\end{array}$ & $\begin{array}{c}0.6305 \\
\mathbf{0 . 0 0 0 0}\end{array}$ & $\begin{array}{c}0.8050 \\
\mathbf{0 . 0 0 0 0}\end{array}$ & 1.0000 & & & \\
\hline $1^{\prime}$ AS & $\begin{array}{c}0.0511 \\
1.0000\end{array}$ & $\begin{array}{l}0.0241 \\
1.0000\end{array}$ & $\begin{array}{c}-0.0139 \\
1.0000\end{array}$ & $\begin{array}{l}0.2640 \\
0.2535\end{array}$ & $\begin{array}{c}0.1553 \\
1.0000\end{array}$ & $\begin{array}{l}0.0923 \\
1.0000\end{array}$ & 1.0000 & & \\
\hline $5^{\prime}$ AS & $\begin{array}{c}-0.0191 \\
1.0000\end{array}$ & $\begin{array}{c}-0.0532 \\
1.0000\end{array}$ & $\begin{array}{c}-0.1143 \\
1.0000\end{array}$ & $\begin{array}{l}0.2620 \\
0.2706\end{array}$ & $\begin{array}{l}0.0673 \\
1.0000\end{array}$ & $\begin{array}{l}0.0366 \\
1.0000\end{array}$ & $\begin{array}{c}0.5430 \\
\mathbf{0 . 0 0 0 0}\end{array}$ & 1.0000 & \\
\hline MV & $\begin{array}{c}-0.1566 \\
1.0000\end{array}$ & $\begin{array}{c}-0.1456 \\
1.0000\end{array}$ & $\begin{array}{l}0.0001 \\
1.0000\end{array}$ & $\begin{array}{c}-0.6106 \\
\mathbf{0 . 0 0 0 0}\end{array}$ & $\begin{array}{c}-0.4697 \\
\mathbf{0 . 0 0 0 0}\end{array}$ & $\begin{array}{c}-0.4204 \\
\mathbf{0 . 0 0 0 4}\end{array}$ & $\begin{array}{c}-0.3535 \\
\mathbf{0 . 0 0 9 0}\end{array}$ & $\begin{array}{c}-0.3494 \\
\mathbf{0 . 0 1 0 7}\end{array}$ & 1.0000 \\
\hline
\end{tabular}

GA: gestational age (days); BW: birth weight (g); HC: head circumference (cm); $1^{\prime}$-AS: 1-minute Apgar score; $5^{\prime}$-AS: 5-minute Apgar score; MV: mechanical ventilation (days). For each cell, the top number corresponds to the correlation coefficient $(R)$ value, whereas the bottom one corresponds to the $p$ value. When correlation is significant, $p$ value is shown in bold.

to assess possible differences in clinical and demographical characteristics between groups.

Possible correlations between variables of interest (i.e., outcome measures) and the other observed variables were studied using the Spearman's rank correlation (Table 3). Gender and $\mathrm{CU}$, being qualitative variables, were excluded from the correlation. However, a possible effect of these latter variables upon outcome measures was studied by means of comparisons between means (one-way analysis of variance, ANOVA) (Table 4), which shows that the only one variable influencing outcome measures is gender.

In addition, by means of a multiple regression analysis an additive effect was found (whilst an interaction effect was excluded) of both gender and treatment upon outcome measures. This multiple regression model has allowed us to test the effects of both treatment, gender being equal, and gender, treatment being equal, upon each of the BSID-II indexes.

Finally, a statistical power analysis was conducted upon the multiple regression analysis. When the power $(1-\beta)$ of the performed test was below the threshold of 0.80 (with $\alpha$ $=0.050)$ the sample size required to putatively achieve the threshold was reported.

Statistical analysis was conducted by F. M., who was kept unaware of the treatment allocation. Analyses were conducted using the Stata 13 statistical software package.

\section{Results}

As can be seen from Table 2, both the intervention group and the control group are matched with respect to gender composition, appropriateness or not of body weight to gestational age, birth weight category, transcranial ultrasound findings,
Apgar scores at $1^{\prime}$ and $5^{\prime}$, and days of assisted ventilation. All these variables, not being related to treatment (as Table 3 shows) but theoretically capable of affecting Bayley's scale scores, can be considered as potential treatment-modifying factors. Conversely, the two groups are not matched with respect to gestational age, birth weight, head circumference, and bronchopulmonary dysplasia. Thus, all of these variables, being related to treatment (as Table 3 shows) and potentially capable of affecting outcome, can be considered as potential confounders. However, there is no reason to believe that these factors are really able to affect the outcome for the following reasons: (a) mean values of gestational age and birth weight remain under 32 weeks and $1500 \mathrm{~g}$, respectively, for both groups and, thus, both groups fall within the limits of age (very preterm) and weight (VLBW) of the neonatal categories which were the object of consideration; (b) different values of head circumference are fully consistent with differences in gestational age and birth weight; and, finally, (c) bronchopulmonary dysplasia is not known from literature as a factor capable of significantly affecting the number of days of assisted ventilation (in turn, days of assisted ventilation were not found capable of affecting outcome, as shown in Table 3).

As Table 3 shows, scores of Bayley's indices are directly related to each other, but they are not related to any of the other variables considered, that is, gestational age, birth weight, head circumference, and days of mechanical ventilation. None of these variables, therefore, affects outcome, and, accordingly, it can be reasonably excluded that they are potential treatment-modifying factors.

As can be seen from Table 4, females of both groups scored higher than males for all three indexes of the Bayley scale. In particular, this difference reached significant values $(p<0.05)$ for PDI and BRS, whilst it only approached to 
significance $(p<0.1)$ for MDI. Therefore, it is plausible to believe that gender is the only one factor that can really affect the outcome.

After having excluded an interaction between gender and treatment, the only predictive model that best fits data collected from our sample is the additive model. This is evident from Figure 1 (plot of means), where outcome measure changes induced by treatment show approximately the same magnitude in both genders. In other words, despite the fact that females always show for all three BSID-II indexes higher values than males, differences between males and females, both in the absence and presence of treatment, remain substantially unchanged. Consequently, the treatment is effective in both groups to the same extent.

In particular, as regards MDI index, gender being equal, the intervention group scored significantly higher mean values than the control group $\left(F_{(1,120)}=8.31 ; p<0.01\right)$, whereas, treatment being equal, females presented higher mean values than males $\left(F_{(1,120)}=5.17 ; p<0.05\right)$.

With regard to PDI index, gender being equal, the intervention group scored higher values than the control group, although this difference was not statistically significant $\left(F_{(1,120)}=2.22 ; p>0.1\right.$, n.s. $)$, whereas, treatment being equal, females presented significantly higher values than males $\left(F_{(1,120)}=4.73 ; p<0.05\right)$.

As regards BRS index, gender being equal, the intervention group scored significantly higher mean values with respect to the control group $\left(F_{(1,120)}=6.93 ; p<0.01\right)$, whereas, treatment being equal, females presented significantly higher mean values than males $\left(F_{(1,120)}=8.85 ; p<\right.$ $0.01)$.

Finally, from the statistical power analysis performed, the high reliability of results relative to index MDI has emerged, since the optimal power level $(1-\beta=0.80)$ has already been reached analyzing 58 cases. On the other hand, only a suboptimal power level has been reached $(1-\beta=0.70)$ for index BRS (to obtain the optimum power of 0.80 at least 80 cases should have been analyzed). Conversely, a sufficient level of power has not been achieved for index PDI. The result must therefore be considered with caution, although it is important to underline that the statistical significance was already obtained with a relatively small number of observations. Moreover, taking into account the fact that the three indexes are highly correlated with each other, there is no reason to believe that PDI and BRS would behave differently from MDI with the increase in the number of observations. It is therefore reasonable to believe that the achievement of a fully reliable result is only a matter of numbers of cases. In fact, to achieve optimal power for PDI at least 257 cases would have to be analyzed. This objective could be realistically achieved by setting up a multicenter study.

\section{Discussion}

4.1. General Findings. Our results are in line with the literature about the effectiveness of early developmental intervention programs focused on the parent-infant relationship and/or infant development in improving overall functional outcomes of preterm infants. In particular, cognitive outcome
TABLE 4: Effects of other demographic and clinical variables (qualitative variables) upon outcome measures.

\begin{tabular}{|c|c|c|c|c|}
\hline Variables & Categories & MDI & PDI & BRS \\
\hline \multirow{3}{*}{ Gender } & Male & $97.75 \pm 2.06$ & $95.31 \pm 1.90$ & $45.89 \pm 4.66$ \\
\hline & Female & $102.82 \pm 1.74$ & $100.39 \pm 1.69$ & $62.69 \pm 4.44$ \\
\hline & p value & 0.062 & 0.048 & 0.011 \\
\hline \multirow{4}{*}{$\mathrm{CU}$} & Normal & $100.4 \pm 1.84$ & $97.56 \pm 1.84$ & $56.11 \pm 4.77$ \\
\hline & Abnormal & $100.61 \pm 2.27$ & $99.74 \pm 2.03$ & $55.82 \pm 35.4$ \\
\hline & Ivh I, II & $99.5 \pm 3.99$ & $95.41 \pm 3.46$ & $46.82 \pm 7.74$ \\
\hline & p value & 0.961 & 0.511 & 0.569 \\
\hline
\end{tabular}

MDI: mental developmental index; PDI: psychomotor developmental index; BRS: behavioural rating scale; CU: cranial ultrasound; Ivh: intraventricular hemorrhage.

For each index, when comparison between categories is significant $p$ value is shown in bold.

turned out to be significantly improved, whereas the motor outcome only slightly changed. This shows that even a homebased program focused on both environmental enrichment and parent-guided environmental interaction, as well as on infant development, may have a comparable effectiveness.

However, in addition, we found a significant improvement in the behavioural outcome. BRS is surprisingly little used in the literature but, on the contrary, would appear to be of some relevance as a measure of adaptation to the environment, at least on a theoretical plane. In our case, it has been able to show a close correspondence between the behavioural approach characterizing the program (i.e., the reciprocal interaction with challenging environments and parents) and the effect of the program itself.

Our results also show that males had significantly lower scores than females either before or after treatment. However, recovery capabilities (meant as the differential gain from baseline) were the same as that of females. In other words, males had the same reactivity to the treatment as females had. Consequently, the treatment was effective in both genders to the same extent.

4.2. Neuroanatomical Correlates of Preterm Birth. In the last decade, it has been shown that preterm born children, even without apparent radiological signs of brain injury, present microstructural damage to the developing white and grey matter that may result in an adverse neurodevelopmental outcome, in both the short and medium-long term [14-17].

In particular, dysmaturation of myelination, axonal density reduction, and impaired dendritic arborization-by impacting projectional, commissural, and association tracts as well as cortical synaptic density - can lead to alterations of neuronal connectivity within and between networks. Consequently, both operation and development of neural networks can result altered [14-17].

4.3. Neuroplasticity and Behavioural (Re)habilitation. The phenomenon of neuroplasticity, that is, the possibility of obtaining changes in the structure or operation of the brain through experience, is potentially/theoretically capable of neutralizing the above-mentioned microstructural damage 


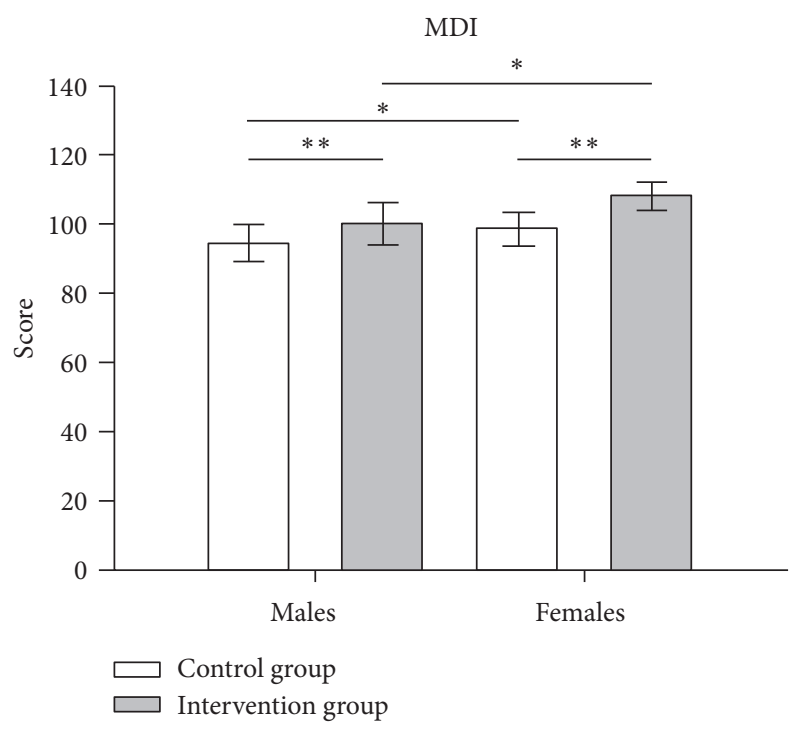

(a)

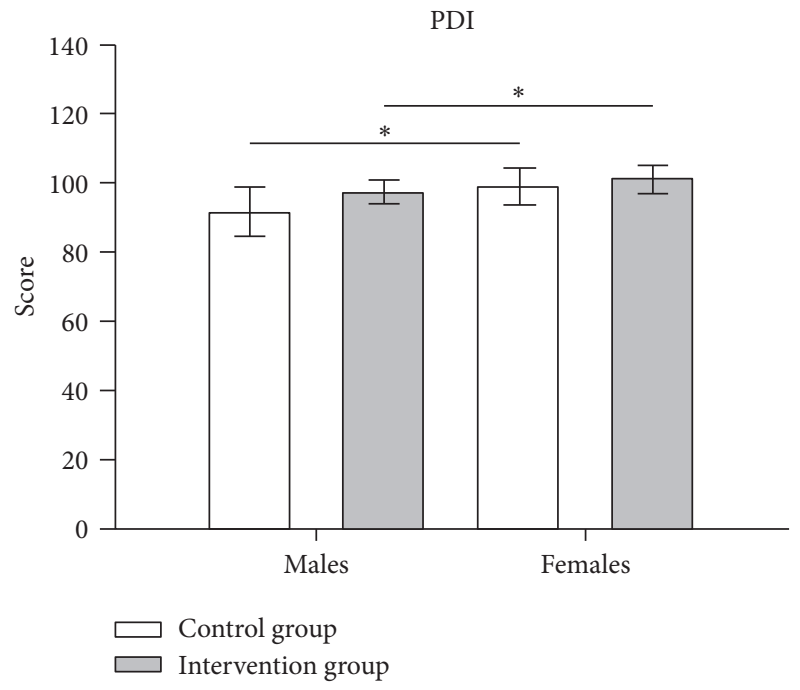

(b)

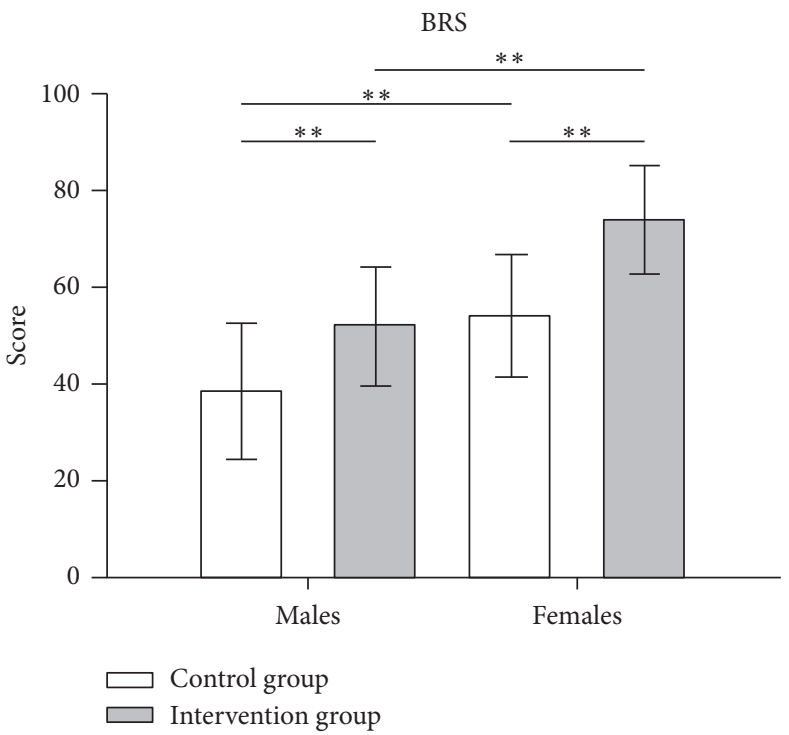

(c)

FIGURE 1: Graphical representation of the multiple regression analysis results for each BSDI II index: (a) mental development index, MDI; (b) psychomotor development index, PDI; and (c) behavioural rating score, BRS. $*$ corresponds to $p<0.05$ and $* *$ to $p<0.01$. Note that indexes changes induced by treatment substantially have the same magnitude in both genders. Consequently, treatment is effective in both genders to the same extent (additive model).

and functional alterations improving the neurodevelopmental outcome of preterm born children. There is, in fact, a two-way relationship between experience and plastic brain changes such that the latter are able, in turn, to produce adaptive behavioural changes. This has led to an increasing development of new strategies for designing both rehabilitation (behavioural rehabilitation) and early intervention programs $[2,18]$.

The working hypothesis is that certain (behavioural) experiences, which have to be both relevant and timely in relation to the individual current developmental stage, are able to revert and/or compensate for the impact of struc- tural/operational alterations through the promotion of brain plasticity, thus increasing the resilience and the development potential of these children. Such experiences would act, therefore, as real neuroprotection factors [19]. In fact, experience-induced changes in gene expression have been proved to alter the production of a variety of proteins, such as those involved in the synthesis of neurotrophic factors (such as NGF and BDNF) known to facilitate both maturation of myelination $[20,21]$ and synaptic plasticity $[2,22-26]$.

In particular, the synthesis and/or the competition of neurotrophic proteins are able to adjust the gain (synaptogenesis) and/or the elimination of synapses (synapse pruning) 
within ensembles of connections, allowing, therefore, the reorganization or reinforcement of synaptic connections in specifically affected neuronal circuits (repairing mechanism) as well as the production of new connections in not directly affected networks (compensatory mechanism). This, substantially, results in a rearrangement of neuronal networks [3], capable, in turn, of fostering adaptive behaviour.

\subsubsection{The Role of Environmental Enrichment and Parent-} Infant Relationship. A factor that has already been pointed out as one of the key features of our program, besides infant development and parent-infant relationship, is environmental enrichment. The ability of environmental enrichment in itself to promote neuronal plasticity has long been known, in both animal models and humans, in either developing, adult or ageing brains (see [19] for review). In particular, environmental enrichment has been shown as being able to enhance maturational processes and reverse cognitive deficits in animal models of chronic perinatal hypoxia (i.e., hypoxicreared mice) [27] as well as to improve motor outcomes in preterm infants at high risk of cerebral palsy $[28,29]$.

However, in our study the environmental element may have acquired an additional role as a reinforcing factor of the two other elements involved, which, in turn, have been proven as effective in promoting development. This is in the context of a synergistic effect where each element of the triad interacts with and facilitates the other.

A timely updated environment to the infant's new needs/requirements could provide the most suitable substrate where the parent-infant relationship can be practised with the ultimate aim/goal of achieving further developmental steps. Upon this common ground both actors can interact: the infant, by making use of a series of inborn skills (such as imitation, shared attention, and empathic understanding), can infer the goal of the observed behaviours and, then, reenact parent's goals and intentions, being capable of receiving step-by-step feedback or feedforward hints (goal-oriented interactive behaviour) [30,31].

According to the concept of metaplasticity, indeed, the potential for interaction of changeable and diversified experiences to broaden out probabilities of plastic changes in the brain is well known $[2,3,32]$. In this context, the achievement of new motor developmental stages has to be meant as an amplification of the capability to interact with the environment. Reciprocally, this triggers a virtuous circle for which the consequent enlargement of related experiences/memories increases the opportunity to recognize challenges that the environment throws up. It is not surprising, in this regard, that in our study functional outcomes mainly improved on a cognitive and adaptive behavioural level $[4,33,34]$.

In conclusion, by considering that especially early experiences have long-lasting effects on later plasticity [3], this kind of program would enable us to meet two theoretical needs of crucial importance: (a) not to deprive infants of the experience needed to create new neural networks, according to the concept of experience-expectant plasticity [3], and, at the same time, (b) to assure infants the most relevant, diverse, and changing (renewing) experiences required to shape the connections of developing or already existing networks, according to the concept of experience-dependent plasticity [3]. Experiences are crucial to allowing infants to recover the development gap and provide, at the same time, the most appropriate basis upon which to build later and more complex development.

4.4. Gender as a Development Modulator. The fact that gender differences exist as regards certain (neuro)developmental outcomes is consistent with the literature [35]. There are, indeed, a growing number of works in favour of a greater vulnerability of males exposed to prenatal and perinatal adversities [36]. Depending on the different studies taken into consideration, this has been attributed to an altered intrauterine metabolic milieu [35], different times of postnatal maturation [37-40], or differences in epigenetic transmission [4143].

Our results show that while males continue to show significantly lower scores than females even after treatment, however their recovery abilities were the same as compared to females. In other words, the nervous central system of males showed the same reactivity to the treatment/development potential as females did. This would seem to suggest the involvement of prenatal causes in determining males' vulnerability.

4.5. Limitations. We feel it necessary, at this point, to review some factors that may have theoretically affected the final effect and in the light of which it is appropriate to consider the results of the present study.

The assignment of children to the study groups was retrospectively made according to whether their parents accepted (the cases) or not (the controls) to take part in the proposed treatment protocol. Those who did not join the program (though adhering to the final evaluation) did so from the beginning and they were then sent to the children's healthcare facilities of reference in order to undertake the care pathway required by law. Participation in the program did not include any costs that families would have to pay to the organizing structure. The only cost to be considered in the broadest sense was the expense in terms of personal commitment and dedicated time. In addition, because there was no difference in the socioeconomic level of the two groups, joining or not joining the program was not influenced by this factor.

Only in 9 out of 70 participants did an interruption of the treatment program occur, and in most cases this was independent of the results obtained and due to various, very different, reasons. These children, however, were not included either in the treatment group or in the control group, precisely in order to obtain the maximum homogeneity of the groups in order not to contaminate data either in one direction or in the other. Therefore, the problem of drop-outs is to be considered quite irrelevant as regards statistical comparisons.

We cannot rule out that the type of assignment on a voluntary basis used in our study did not indirectly select for the treatment group families with a higher perception of clinical risk and/or a greater awareness and care for the needs of their children. On the other hand, the highly motivated participation of parents in the protocol can be seen as part 
of the treatment itself and as an effect catalyst, rather than a mere statistical bias.

Indeed, it is well known that both parental care and interaction with parents, even for nonprimate mammals, as a primary core of social relations, are part of the issue of enrichment of the environment and contribute in themselves to the promotion of neural plasticity $[44,45]$. Apart from this, however, it can be assumed that the assignment of the children, on whom the effect of treatment was measured, to the study groups occurred randomly, no one having been directly selected.

In the present work, children with a high risk of developing major disabilities due to the presence of ascertained brain lesions (by means of cranial ultrasound and/or magnetic resonance imaging) were not taken into account. Their inclusion could have entailed assembling subgroups with a different response capability to the enriched environment from the very beginning, because of the more severe brain damage. These children were followed up at our institution until up to three months of corrected age; then, according to normal practice, they were entrusted to the children's neuropsychiatric medical centres, competent for the area, so that a specific and diversified rehabilitation program was started.

The exclusion from the study protocol of high-risk children, accordingly, allowed us to verify the effect of the enriched environment on more homogeneous study groups both in terms of type of perinatal brain damage and, consequently, of starting anatomical-functional substrate (within and between groups), and type of treatment (within groups). In any case, the treated group showed a better outcome than the nontreated.

We cannot rule out that other factors did not influence the final outcome by acting as potential confounders (parents' personality or mental health or even couple dynamics rather than environmental factors, etc.) but these factors were not taken into consideration at the time of data acquisition and it is upon already acquired documentary data that a retrospective study is conducted. We believe, however, that this may be a more appropriate topic for specific epidemiological studies and, therefore, in part falls outside the purposes of our study.

None of the children who participated in our study presented major disabilities at the endpoint. This could be partly due to the exclusion from the study of high-risk children and partly from having taken into consideration a period that was too short to detect their impact, although the period of time used is the time most frequently used according to the literature [8].

Finally, in the present work an outdated version of the Bayley scale was used because it was the only one available at the time of data collection. This version, however, compared to the current one, has the advantage of considering the BRS index, particularly suitable for estimating the behavioural effects of the treatment and, therefore, in line with the primary aim of our study.

4.6. Concluding Remarks. In recent years we have witnessed a progressive growth of clinical studies aimed at testing the effectiveness of early developmental intervention programs in improving overall functional outcomes of preterm born children. Nevertheless, or precisely for this reason, such studies are somewhat heterogeneous about either the different approaches used or the preset goals, thus limiting to some extent the conclusions that can be drawn.

This requires that further efforts are made to especially understand what are the most effective components in promoting neural plasticity and, therefore, outcomes in multifactorial programs and what the best combinations between them are, also with the aim/intention of optimizing the balance between health resources and results.

In this regard, the present program certainly appears highly cost-effective, taking into account its characteristics of being both home-based and family-centred. This could make it an effective prevention tool thoroughly adoptable as part of a neuroprotective strategy for very preterm born infants, particularly in low- and middle-income countries.

\section{Competing Interests}

The authors declare that there is no conflict of interests regarding the publication of this paper.

\section{Acknowledgments}

The authors wish to thank Professor John Ayers for language revision.

\section{References}

[1] H. Blencowe, A. C. C. Lee, S. Cousens et al., "Preterm birth-associated neurodevelopmental impairment estimates at regional and global levels for 2010," Pediatric Research, vol. 74, supplement 1, pp. 17-34, 2013.

[2] B. Kolb and A. Muhammad, "Harnessing the power of neuroplasticity for intervention," Frontiers in Human Neuroscience, vol. 8, article 377, 2014.

[3] B. Kolb and R. Gibb, "Searching for the principles of brain plasticity and behavior," Cortex, vol. 58, pp. 251-260, 2014.

[4] B. Kolb, R. Mychasiuk, A. Muhammad, Y. Li, D. O. Frost, and R. Gibb, "Experience and the developing prefrontal cortex," Proceedings of the National Academy of Sciences of the United States of America, vol. 109, S2, pp. 17186-17193, 2012.

[5] G. McAnulty, F. Duffy, S. Kosta et al., "School age effects of the Newborn Individualized Developmental Care and Assessment Program for medically low-risk preterm infants: Preliminary findings," Journal of Clinical Neonatology, vol. 1, no. 4, pp. 184194, 2012.

[6] M. V. Johnston, A. Nishimura, K. Harum, J. Pekar, and M. E. Blue, "Sculpting the developing brain," Advances in Pediatrics, vol. 48, pp. 1-38, 2001.

[7] H. Als, F. H. Duffy, G. B. McAnulty et al., "Early experience alters brain function and structure," Pediatrics, vol. 113, no. 4, pp. 846-857, 2004.

[8] A. Spittle, J. Orton, P. Anderson, R. Boyd, and L. W. Doyle, "Early developmental intervention programmes post-hospital discharge to prevent motor and cognitive impairments in preterm infants," Cochrane Database of Systematic Reviews, vol. 12, Article ID CD005495, 2012.

[9] J. A. Vanderveen, D. Bassler, C. M. T. Robertson, and H. Kirpalani, "Early interventions involving parents to improve 
neurodevelopmental outcomes of premature infants: a metaanalysis," Journal of Perinatology, vol. 29, no. 5, pp. 343-351, 2009.

[10] N. Bayley, Bayley Scales of Infant Development, The Psychological Corporation, San Antonio, Tex, USa, 2nd edition, 1993.

[11] H. Als, "NIDCAP: testing the effectiveness of a relationshipbased comprehensive intervention," Pediatrics, vol. 124 , no. 4 , pp. 1208-1210, 2009.

[12] A. Ohlsson and S. E. Jacobs, "NIDCap: a systematic review and meta-analyses of randomized controlled trials," Pediatrics, vol. 131, no. 3, pp. e881-e893, 2013.

[13] H. Als, "Toward a synactive theory of development: promise for the assessment and support of infant individuality," Infant Mental Health Journal, vol. 3, no. 4, pp. 229-243, 1982.

[14] S. Groeschel, J.-D. Tournier, G. B. Northam et al., "Identification and interpretation of microstructural abnormalities in motor pathways in adolescents born preterm," NeuroImage, vol. 87, pp. 209-219, 2014.

[15] L. R. Ment, D. Hirtz, and P. S. Hüppi, "Imaging biomarkers of outcome in the developing preterm brain," The Lancet Neurology, vol. 8, no. 11, pp. 1042-1055, 2009.

[16] J. Skranes, T. R. Vangberg, S. Kulseng et al., "Clinical findings and white matter abnormalities seen on diffusion tensor imaging in adolescents with very low birth weight," Brain, vol. 130, no. 3, pp. 654-666, 2007.

[17] G. B. Northam, F. Liégeois, W. K. Chong, J. S. Wyatt, and T. Baldeweg, "Total brain white matter is a major determinant of IQ in adolescents born preterm," Annals of Neurology, vol. 69, no. 4, pp. 702-711, 2011.

[18] S. C. Cramer, M. Sur, B. H. Dobkin et al., "Harnessing neuroplasticity for clinical applications," Brain, vol. 134, no. 6, pp. 1591-1609, 2011.

[19] A. Sale, N. Berardi, and L. Maffei, "Environment and brain plasticity: towards an endogenous pharmacotherapy," Physiological Reviews, vol. 94, no. 1, pp. 189-234, 2014.

[20] J. Xiao, T. J. Kilpatrick, and S. S. Murray, "The role of neurotrophins in the regulation of myelin development," NeuroSignals, vol. 17, no. 4, pp. 265-276, 2009.

[21] J. Xiao, A. W. Wong, M. M. Willingham, M. Van Den Buuse, T. J. Kilpatrick, and S. S. Murray, "Brain-derived neurotrophic factor promotes central nervous system myelination via a direct effect upon oligodendrocytes," NeuroSignals, vol. 18, no. 3, pp. 186-202, 2011.

[22] H. Park and M.-M. Poo, "Neurotrophin regulation of neural circuit development and function," Nature Reviews. Neuroscience, vol. 14, no. 1, pp. 7-23, 2013.

[23] F. Gelfo, D. Cutuli, F. Foti et al., "Enriched environment improves motor function and increases neurotrophins in hemicerebellar lesioned rats," Neurorehabilitation and Neural Repair, vol. 25, no. 3, pp. 243-252, 2011.

[24] B. B. Johansson, "Brain plasticity and stroke rehabilitation. The Willis lecture," Stroke, vol. 31, no. 1, pp. 223-230, 2000.

[25] S. A. Back, "Cerebral white and gray matter injury in newborns: new insights into pathophysiology and management," Clinics in Perinatology, vol. 41, no. 1, pp. 1-24, 2014.

[26] J. M. Dean, L. Bennet, S. A. Back, E. McClendon, A. Riddle, and A. J. Gunn, "What brakes the preterm brain? An arresting story," Pediatric Research, vol. 75, no. 1-2, pp. 227-233, 2014.

[27] N. Salmaso, S. Tomasi, and F. M. Vaccarino, "Neurogenesis and maturation in neonatal brain injury," Clinics in Perinatology, vol. 41, no. 1, pp. 229-239, 2014.
[28] C. Morgan, I. Novak, and N. Badawi, "Enriched environments and motor outcomes in cerebral palsy: systematic review and meta-analysis," Pediatrics, vol. 132, no. 3, pp. e735-e746, 2013.

[29] C. Morgan, I. Novak, R. C. Dale, and N. Badawi, "Optimising motor learning in infants at high risk of cerebral palsy: a pilot study," BMC Pediatrics, vol. 15, no. 1, article 30, 2015.

[30] A. N. Meltzoff, P. K. Kuril, J. Movellan, and T. J. Sejnowski, "Foundations for a new science of learning," Science, vol. 325, no. 5938, pp. 284-288, 2009.

[31] P. Ruvolo, D. Messinger, and J. Movellan, "Infants time their smiles to make their moms smile," PLOS ONE, vol. 10, no. 9, Article ID e0136492, 2015.

[32] W. C. Abraham and M. F. Bear, "Metaplasticity: the plasticity of synaptic plasticity," Trends in Neurosciences, vol. 19, no. 4, pp. 126-130, 1996.

[33] M. Ploughman, "Exercise is brain food: the effects of physical activity on cognitive function," Developmental Neurorehabilitation, vol. 11, no. 3, pp. 236-240, 2008.

[34] K. M. Langa and D. A. Levine, "The diagnosis and management of mild cognitive impairment: a clinical review," The Journal of the American Medical Association, vol. 312, no. 23, pp. 2551-2561, 2014.

[35] A. Spinillo, L. Montanari, B. Gardella, M. Roccio, M. Stronati, and E. Fazzi, "Infant sex, obstetric risk factors, and 2-year neurodevelopmental outcome among preterm infants," Developmental Medicine and Child Neurology, vol. 51, no. 7, pp. 518-525, 2009.

[36] J. A. DiPietro and K. M. Voegtline, "The gestational foundation of sex differences in development and vulnerability," Neuroscience, 2015.

[37] M. Thordstein, N. Löfgren, A. Flisberg, K. Lindecrantz, and I. Kjellmer, "Sex differences in electrocortical activity in human neonates," NeuroReport, vol. 17, no. 11, pp. 1165-1168, 2006.

[38] J. A. DiPietro, K. T. Kivlighan, K. A. Costigan et al., "Prenatal antecedents of newborn neurological maturation," Child Development, vol. 81, no. 1, pp. 115-130, 2010.

[39] C. A. Malcolm, D. L. McCulloch, and A. J. Shepherd, "Patternreversal visual evoked potentials in infants: gender differences during early visual maturation," Developmental Medicine and Child Neurology, vol. 44, no. 5, pp. 345-351, 2002.

[40] R. Achiron, S. Lipitz, and A. Achiron, "Sex-related differences in the development of the human fetal corpus callosum: in utero ultrasonographic study," Prenatal Diagnosis, vol. 21, no. 2, pp. 116-120, 2001.

[41] A. Gabory, L. Attig, and C. Junien, "Sexual dimorphism in environmental epigenetic programming," Molecular and Cellular Endocrinology, vol. 304, no. 1-2, pp. 8-18, 2009.

[42] T. C. Ngun, N. Ghahramani, F. J. Sánchez, S. Bocklandt, and E. Vilain, "The genetics of sex differences in brain and behavior," Frontiers in Neuroendocrinology, vol. 32, no. 2, pp. 227-246, 2011.

[43] M. M. McCarthy, A. P. Auger, T. L. Bale et al., "The epigenetics of sex differences in the brain," Journal of Neuroscience, vol. 29, no. 41, pp. 12815-12823, 2009.

[44] B. L. Callaghan and N. Tottenham, "The neuro-environmental loop of plasticity: a cross-species analysis of parental effects on emotion circuitry development following typical and adverse caregiving," Neuropsychopharmacology, vol. 41, no. 1, pp. 163176, 2016.

[45] R. L. Holt and M. A. Mikati, "Care for child development: basic science rationale and effects of interventions," Pediatric Neurology, vol. 44, no. 4, pp. 239-253, 2011. 

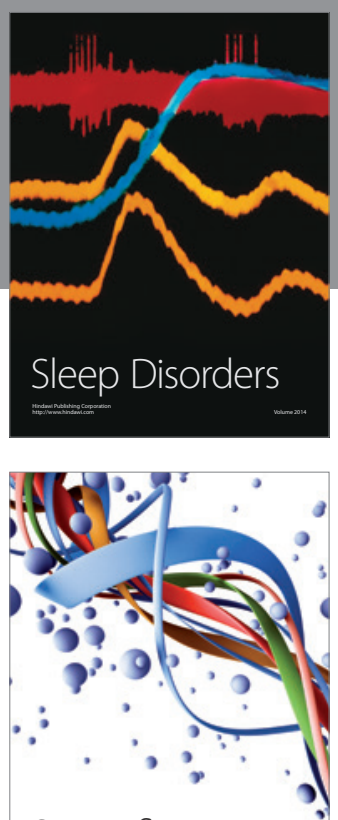

Scientifica
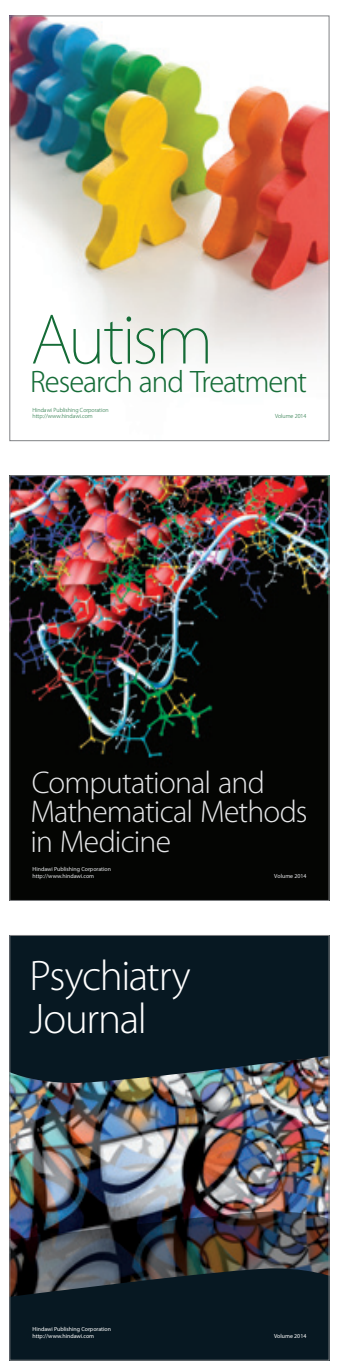
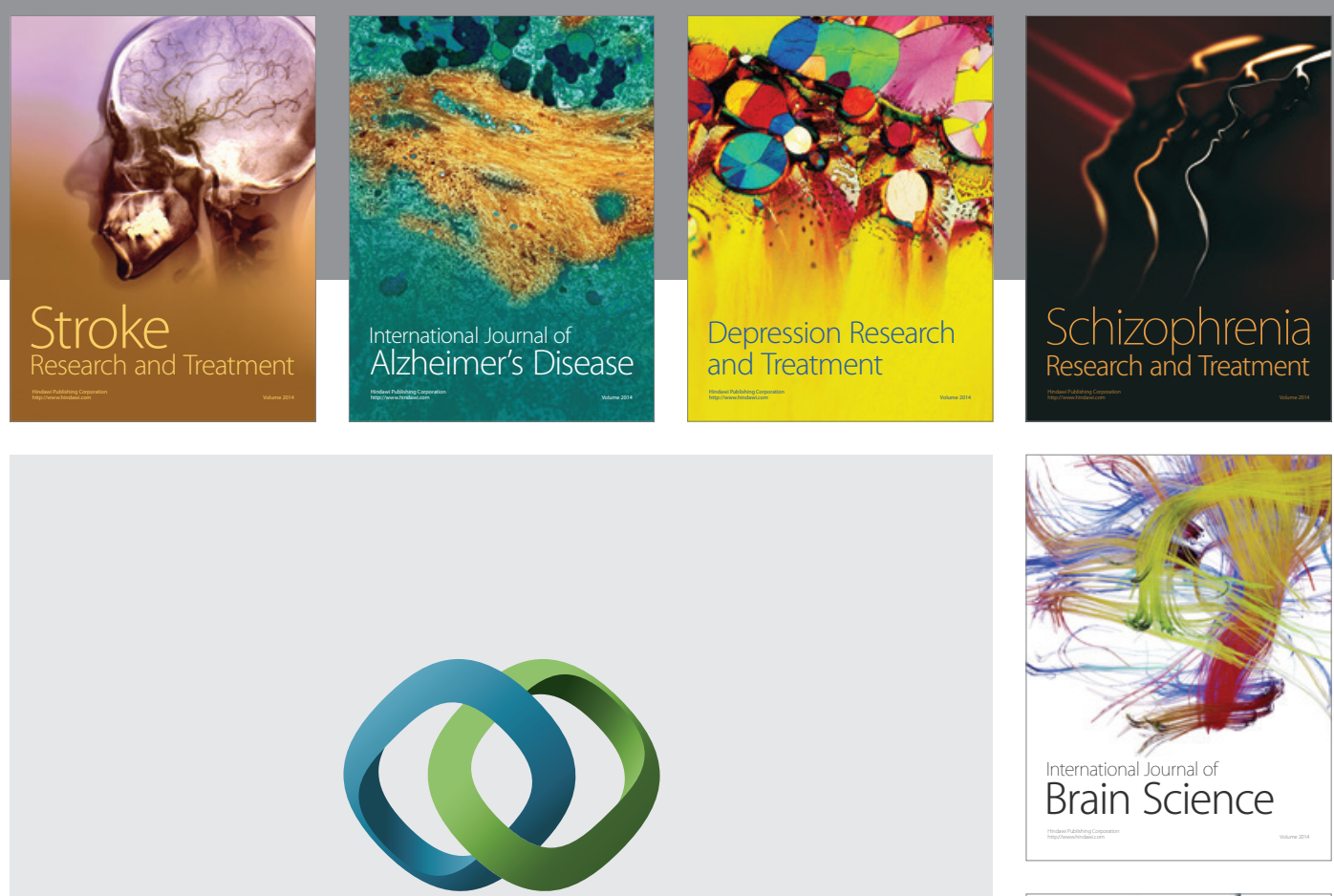

\section{Hindawi}

Submit your manuscripts at

http://www.hindawi.com
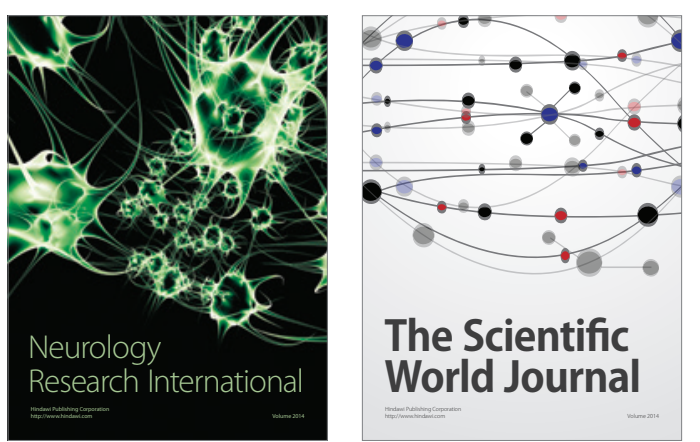

The Scientific World Journal

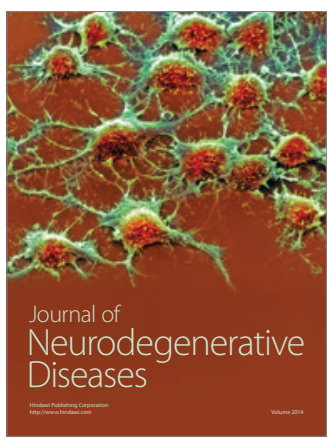

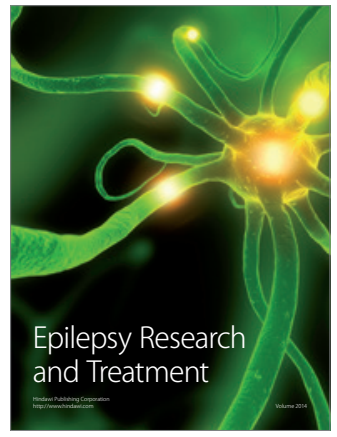

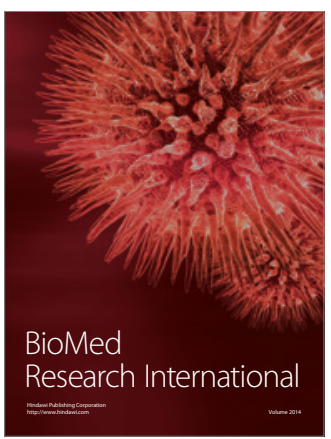

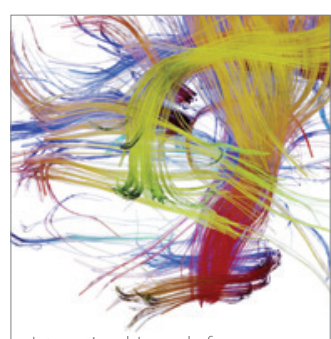

Brain Science

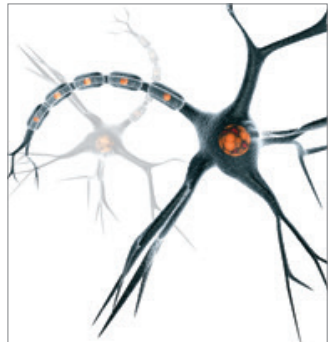

Neural Plasticity
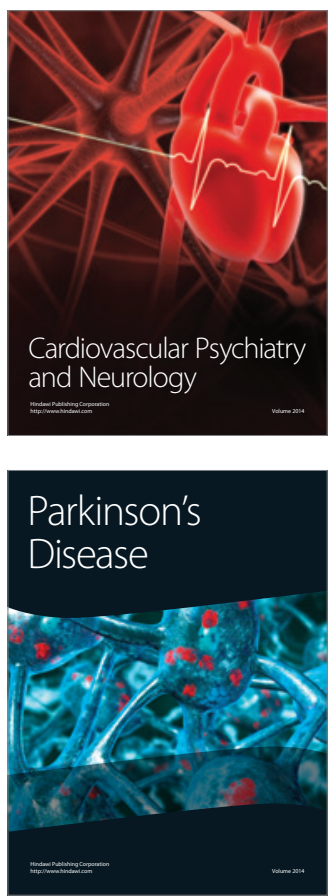